\title{
Where are the beachmasters? Unexpectedly weak polygyny among southern elephant seals on a South Shetland Island
}

\author{
H. J. Nichols ${ }^{1,2}$ \\ J. I. Hoffman ${ }^{2, *}$ \\ B. Fuchs ${ }^{2}$, A. J. Paijmans ${ }^{2}$ \\ G. Lewis ${ }^{1}$, \\ C. A. Bonin ${ }^{3}$ \\ M. E. Goebel ${ }^{4, *, t}$ \\ \& \\ ${ }^{1}$ Department of Biosciences, Swansea University, Swansea, UK \\ ${ }^{2}$ Department of Animal Behaviour, University of Bielefeld, Bielefeld, Germany \\ ${ }^{3}$ Department of Marine and Environmental Sciences, Hampton University, Hampton, USA \\ ${ }^{4}$ Institute of Marine Sciences, University of California Santa Cruz, Santa Cruz, CA, USA
}

\section{Keywords}

beachmaster; mating system plasticity; Mirounga leonina; polygyny; reproductive skew; Southern elephant seal.

\section{Correspondence}

Hazel J. Nichols, Department of Biosciences, Swansea University, Singleton Park, Swansea SA2 8PP, UK.

Email: H.J.Nichols@swansea.ac.uk

\section{Editor: Nigel Bennett}

* Joint senior authors.

${ }^{\dagger}$ Former address: Antarctic Ecosystem Research Division, Southwest Fisheries Science Center, NOAA/NMFS, La Jolla, California, USA.

Received 11 January 2021; revised 30 September 2021; accepted 4 October 2021

doi:10.1111/jzo.12936

\begin{abstract}
Intraspecific variation in animal mating systems can have important implications for ecological, evolutionary and demographic processes in wild populations. For example, patterns of mating can impact social structure, dispersal, effective population size and inbreeding. However, few species have been studied in sufficient detail to elucidate mating system plasticity and its dependence on ecological and demographic factors. Southern elephant seals (Mirounga leonina) have long been regarded as a textbook example of a polygynous mating system, with dominant 'beachmaster' males controlling harems of up to several hundred females. However, behavioural and genetic studies have uncovered appreciable geographic variation in the strength of polygyny among elephant seal populations. We, therefore, used molecular parentage analysis to investigate patterns of parentage in a small satellite colony of elephant seals at the South Shetland Islands. We hypothesised that dominant males would be able to successfully monopolise the relatively small numbers of females present in the colony, leading to relatively high levels of polygyny. A total of 424 individuals (comprising 33 adult males, 101 adult females and 290 pups) sampled over 8 years were genotyped at 20 microsatellites and reproductive success was analysed by genetically assigning parents. Paternity could only be assigned to 31 pups (10.7\%), despite our panel of genetic markers being highly informative and the genotyping error rate being very low. The strength of inferred polygyny was weak in comparison to previous genetic studies of the same species, with the most successful male fathering only seven pups over the entire course of our study. Our results show that, even in a species long regarded as a model for extreme polygyny, male reproductive skew can vary substantially among populations.
\end{abstract}

\section{Introduction}

Understanding mating systems and their evolution is a central goal of behavioural and molecular ecology, animal behaviour and evolutionary biology (Clutton-Brock, 1989; Kempenaers, 2008; Neff \& Pitcher, 2005; Reynolds, 1996). Mating systems influence a multitude of ecological and evolutionary processes, ranging from social structure, dispersal and gene flow through to the evolution of life-history and sexually selected traits, local adaptation and ultimately speciation (Dieckmann et al., 1999; Ross, 2001). Understanding mating systems can also have practical implications because of their downstream impacts on inbreeding, effective population size variation and population dynamics, which can influence the ability of populations to respond to challenges such as environmental change
(Nunney, 1993; Plesnar-Bielak et al., 2012; Waser et al., 1986).

Most mammalian mating systems are characterised by unequal investment between the sexes in reproduction (Trivers, 1972). As a result, males usually compete with one another to mate with as many females as possible, whereas females are often choosy, selecting males who can provide them with either direct or indirect fitness benefits (Fisher, 1958; Prokop et al., 2012; Zahavi, 1975). Sexual selection for indicators of male fitness such as large body size, behavioural dominance and the control of resources results in polygynous mating systems, where the variance in reproductive success among males of the same population can be considerable (Clutton-Brock, 1988). The extent to which male reproductive success varies among species depends on ecological and phylogenetic factors 
that determine the distribution of oestrus females over space and time (Emlen \& Oring, 1977), including the numbers of adult males and females present in breeding groups (Kutsukake \& Nunn, 2006).

Further complexity arises from the observation that mating systems not only differ among species, but can also vary within species. This is in accordance with theoretical discussions emphasising the importance of ecological and demographic factors shaping the ability of males to gain access to females or the resources required to attract them (Emlen \& Oring, 1977). Reproductive skew can also be influenced by the costs and benefits that individuals experience of breeding together and the tactics that competitors use to maximise their own reproductive success (Clutton-Brock, 2016; Hodge, 2009). However, studies of intraspecific variation in mating systems are relatively uncommon and mating systems are often considered as more-or-less fixed attributes of a given species (Gursky-Doyen, 2010). Consequently, more studies of intraspecific variation in mating systems are needed both to gain a broader understanding of the magnitude of variation within versus among species and to understand the specific factors responsible for that variation.

The southern elephant seal (Mirounga leonina) is a textbook example of a strongly polygynous mammal (Clutton-Brock, 2016) that provides an excellent opportunity to investigate variation in animal mating systems. Southern elephant seals spend most of their lives foraging at sea but return to terrestrial haul-out sites to breed during the austral summer months. Although foraging often takes place far away from these sites, maternally directed natal philopatry tends to limit the exchange of individuals among breeding colonies (Nichols, 2009). Consequently, four main, genetically distinct populations have been recognised: South Georgia in the South Atlantic Ocean, Heard and Kerguelen Islands in the South Indian Ocean, Macquarie Island in the South Pacific Ocean, and the Península Valdés population on the coast of mainland Argentina (Hoelzel et al., 2001; McMahon et al., 2005; Slade et al., 1998). While gene flow between these populations is rare, dispersal may occur more frequently between some subpopulations. For example, tagging and satellite tracking studies show evidence of dispersal of both sexes between Marion Island, Iles Crozet and Iles Kerguelen (within the Kerguelen population; Oosthuizen et al., 2011).

Southern elephant seals exhibit extreme sexual size dimorphism, with males being approximately six times larger than females (González-Suárez and Cassini, 2014). During the breeding season, females congregate on beaches to pup and remate, and dominant males (known as a 'beachmasters') fight to control harems of females (defined as a minimum of two females with a dominant male in attendance). Behavioural studies at South Georgia (Laws, 1956; McCann, 1980; Modig, 1996), the Falkland Islands (Galimberti et al., 2002), Marion Island (Wilkinson \& van Aarde, 1999) and Macquarie Island (Carrick \& Ingham, 1962) have shown that a handful of the highest ranking males can monopolise harems of many tens to over a thousand breeding females on densely packed beaches. The largest known aggregation was 1600 females, observed on Heard Island in October 1985 (Bester, 2014), with 22 males in attendance (M.N. Bester pers obs). Dominant males tend to be older and larger than subordinate males, who attempt to gain mating opportunities by entering harems to secure 'sneaky' matings, intercepting females as they leave the harems to forage at sea (McCann, 1981), and potentially by mating with females at sea (De Bruyn et al., 2011). Although these alternative mating strategies do appear to have a limited payoff for subordinate males, genetic studies of this species have shown that behavioural observations generally provide a reasonable proxy of male reproductive success, with harem holders typically accounting for a large proportion (up to 90\%) of all paternities (Fabiani et al., 2004; Hoelzel et al., 1999; Wainstein et al., 1997). Furthermore, beachmasters have been known to hold harems over multiple consecutive years (Fabiani et al., 2004), which could result in the most successful males fathering hundreds of pups over their lifespans.

However, polygyny need not always be this extreme in elephant seals. For example, southern elephant seals on the Península Valdés breed at relatively low density due to the availability of hundreds of kilometres of uninterrupted, open beaches. This results in harems that are on average smaller than those observed at other localities (median $=11$ females per harem, range 2-122) within which females are spaced out (e.g. single harems of over 100 females take up at least $12000 \mathrm{~m}^{2}$ of beach), making it difficult for harem-holding males to monopolise matings (Baldi et al., 1996). For example, dominant males with large harems (over 50 females) have a high number of copulations (37 per $100 \mathrm{~h}$ ), but these harems are difficult to control by a single male and females frequently mate with subordinate males ( 9 copulations per $100 \mathrm{~h}$; Baldi et al., 1996). While the degree of polygyny on the Península Valdés is lower than at high density breeding colonies, harem holders still have high reproductive success, achieving $55 \%$ of observed copulations and $58 \%$ of paternities (Hoelzel et al., 1999), while $75 \%$ of males father no offspring (Wainstein, 2000).

The most southerly breeding sites for southern elephant seals are located in the South Orkney Islands and the South Shetland Islands (Laws, 1956). These populations could potentially have different patterns of male reproductive skew than previously studied populations for two main reasons. First, at Signy Island in the South Orkneys, around 70 pups per year were recorded as having been born in 4-6 harems (Laws, 1956). These small harem sizes will limit the maximal reproductive success of harem holders simply because these males will have access to fewer breeding females. Second, in particularly cold years, breeding takes place on fast ice where space is unrestricted, leading to even greater female dispersion and potentially lower male reproductive skew (Laws, 1956). However, genetic studies have not been conducted in these localities, so the realised degree of polygyny is unknown. Conversely, smaller harems might in fact be easier for dominant males to monopolise, allowing beachmasters to attain relatively high reproductive success at low population densities. This was previously shown for a low density colony of Antarctic fur seals in the South Shetlands, where the most successful males surpassed the reproductive success of dominant males at a high density colony in South Georgia (Bonin et al., 2014). Genetic 
studies are, therefore, required to assess the degree of reproductive skew in lower density colonies of southern elephant seals and to further understand the drivers of variation in polygyny.

Here, we use genetic parentage analysis to investigate the degree of polygyny of southern elephant seals at Half Moon Beach, Cape Shirreff, in the South Shetland Islands (Fig. 1). The relatively small Cape Shirreff breeding colony is considered a 'satellite' to South Georgia because there is some exchange of individuals between these locations (Boyd et al., 1996). Together with other islands in the archipelago, such as King George Island and Elephant Island, Cape Shirreff may represent a stop-off point for breeding females migrating northwards to larger colonies from southerly pelagic foraging grounds closer to the Antarctic Front, with some females remaining there to breed (Krzemiński, 1981; Laws, 1994). In warmer, more favourable years, many females breed at Cape Shirreff, whereas in colder, less favourable years, the accumulation of sea ice and snow causes fewer animals to come ashore. Consequently, the size of the breeding population varies appreciably from year to year. Similar to the Península Valdés, the beach at Half Moon Bay represents a large expanse of uninterrupted breeding habitat that is sparsely occupied by southern elephant seals in the breeding season. In most years, there is only one harem, but in years of high pup production there can be as many as five harems.

In order to investigate the strength of polygyny in this breeding colony, we sampled all of the pups born across eight breeding seasons, together with the majority of adult males and a large number of breeding females. To maximise the power to resolve parental relationships, we genotyped all of the individuals at 20 highly variable microsatellites. Wherever possible, maternities were assigned in order to maximise the power of the paternity analysis and to provide a benchmark against that patterns of male reproductive success can be compared. As previous studies of Antarctic fur seals have shown that male reproductive skew is higher at low density (Bonin et al., 2014; Hoffman et al., 2003), we hypothesised that the mating system of southern elephant seals at Cape Shirreff would be highly polygynous, with a small proportion of males monopolising reproductive success. Both the degree of reproductive skew between competing males and the size of the harem they compete over are likely to contribute to dominant male reproductive success. We, therefore, also predicted that the maximum annual reproductive success of harem holders may be lower at Cape Shirreff than at high density breeding sites, due to the smaller sizes of the harems.

\section{Materials and methods}

\section{Study site and sample collection}

This study was conducted at a small breeding colony at Half Moon Beach, Cape Shirreff, South Shetland Islands $\left(62^{\circ} 28^{\prime} 37.4^{\prime \prime}, 60^{\circ} 46^{\prime} 45.0^{\prime \prime}\right.$, Figure 1). Cape Shirreff is an icefree peninsula, covering approximately $3.1 \mathrm{~km}^{2}$. It is the most northerly point on the north coast of Livingston Island. Half Moon Beach is a horseshoe shaped beach stretching over $1.65 \mathrm{~km}$. It is a wide-open sandy/cobble beach with areas of higher elevation that contain a few large boulders and one long rocky outcrop extending perpendicularly relative to the waterline and located approximately $70 \mathrm{~m}$ from the low tide line. Elephant seals tend to concentrate around these areas of the beach, typically in a single harem but sometimes in as many as five, which are separated by as much as $400 \mathrm{~m}$ (Fig. 2). During the breeding season (October-November), pupping areas are covered with between a few centimetres and $2 \mathrm{~m}$ of snow. Access to harems often requires navigating a substantial berm of ice and snow at the high tide mark. Due to the remote setting and challenging conditions, researchers typically arrive at Cape Shirreff midway through pupping (mean arrival date 29 October, $\mathrm{SD}=7.3$ days), leading to the inference that the southern elephant seals start hauling out at the breeding site in early October. The mean number of animals present during the
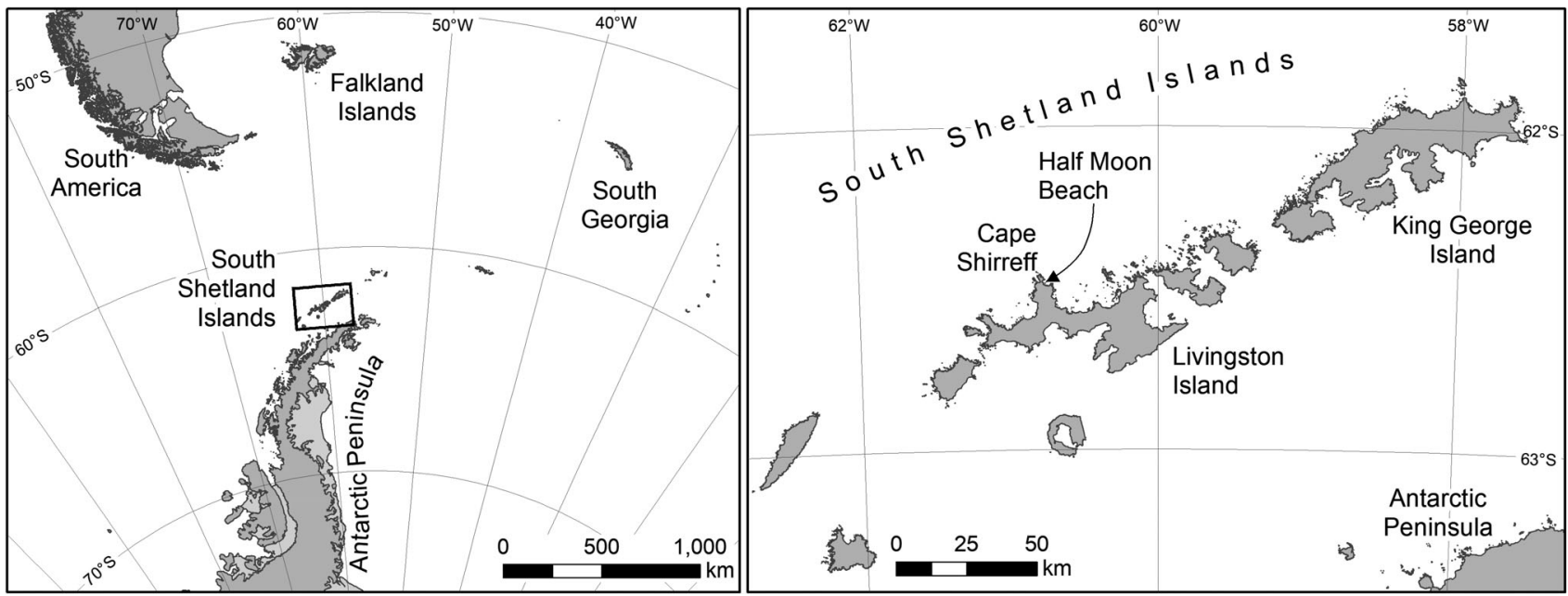

Figure 1 The study location and terrain at Half Moon beach, Cape Shirreff, in the South Shetland Islands. 
breeding season in our study was $106(\mathrm{SD}=37.7$; range $=56$ 158). As in other populations, harems were dominated by a single male, with subordinate males present on the periphery or elsewhere on the beach. Approximately $95 \%$ of adult males, including all harem holders and the majority of peripheral males were sampled together with all of the pups and females that were present on the beach. Individuals were carefully approached at the harems in order to minimise disturbance.

Fieldwork for this project was conducted during the Austral spring (October to December inclusive) of 2008 to 2016, except for 2010 (Table S1). Samples were collected during 2010 but unfortunately these were lost in transit from Cape Shirreff back to the USA. In most seasons, adults were sampled on the same day, but when the numbers of individuals were large, they were sampled by harem over consecutive days. Adults were sampled from the flanks using a $2 \mathrm{~mm}$ sterile, disposable Miltex ${ }^{\mathrm{TM}}$ biopsy punch (Fisher Scientific). Pup skin samples were collected from a rear flipper while they were captured for tagging using a tag hole punch or a $2 \mathrm{~mm}$, sterile, disposable Miltex biopsy punch. Skin samples were immediately placed in $95 \%$ ETOH and kept frozen $\left(-20^{\circ} \mathrm{C}\right)$ until analysis. Sampled adults were marked using hair dye whenever the bulk of sampling efforts spanned more than a day (sampled individuals were otherwise identified by their biopsy punch mark). Marks persisted throughout the season but were lost at the moult so were not informative from one season to the next. Most individuals were sampled within a few days of the first visit to the colony but the site was checked for unsampled individuals thereafter during broad pinniped censuses at Cape Shirreff and / or to retrieve instrumentation.

\section{Microsatellite genotyping}

The DNA was extracted using a standard chloroform/isoamyl alcohol protocol (Sambrook et al., 1989) and genotyped at 20 polymorphic microsatellites (see Table S1 for details). PCR amplifications were performed in five separate multiplexed reactions using a Qiagen ${ }^{\circledR}$ Multiplex PCR Kit following the manufacturer's recommendations, except that we used $12 \mu 1$ reaction volumes to keep the use of reagents to a minimum. The following PCR profile was used: one cycle of $5 \mathrm{~min}$ at $94^{\circ} \mathrm{C} ; 24$ cycles of $30 \mathrm{~s}$ at $94^{\circ} \mathrm{C}, 90 \mathrm{~s}$ at $60^{\circ} \mathrm{C}, 30 \mathrm{~s}$ at $72^{\circ} \mathrm{C}$; and one final cycle of $15 \mathrm{~min}$ at $72^{\circ} \mathrm{C}$. Between 12 and 20 positive controls were included on each 96-well PCR plate to facilitate the standardisation of microsatellite scoring across
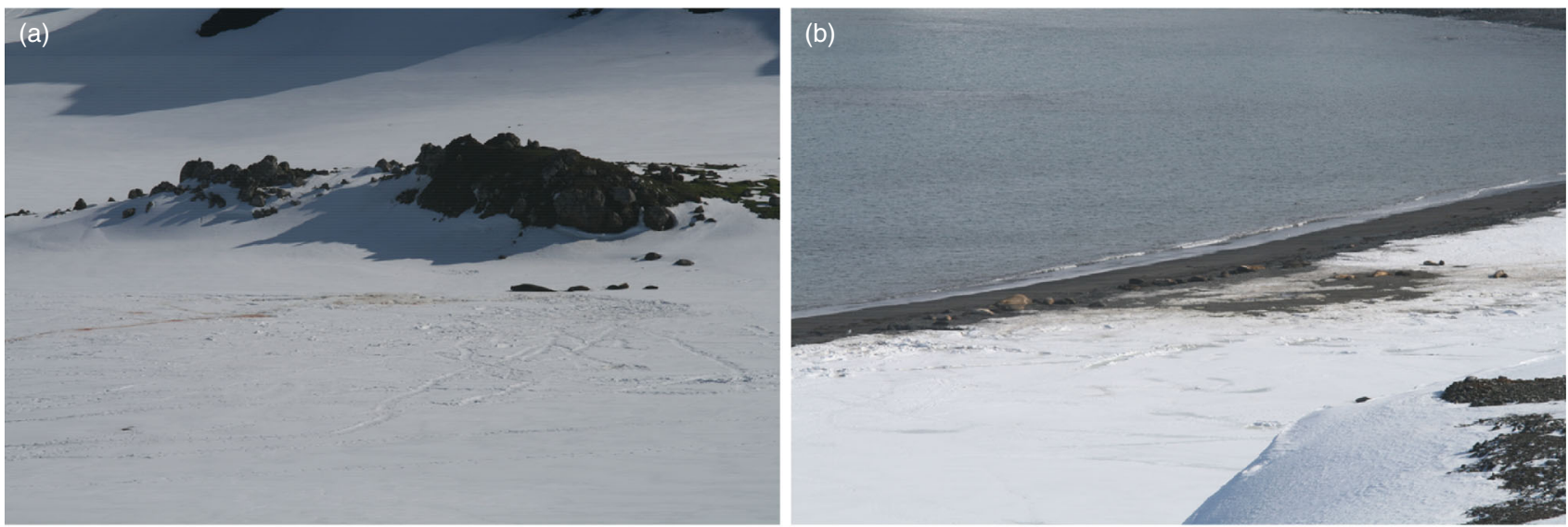

(c)

(d)
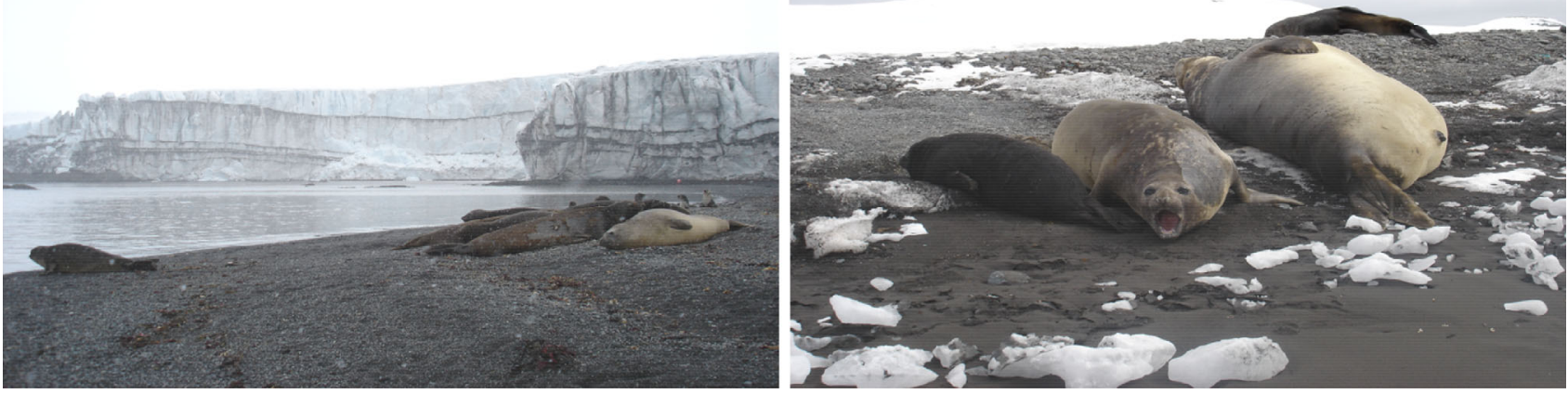

Figure 2 Southern elephant seal harems at Half Moon beach, showing (a) a small harem located close to an elevated rocky area that could potentially provide cover for males pursuing alternative mating tactics, ( $b$ and $c$ ) harems close to the shoreline at high tide, and (d) a female (centre) with her pup and a harem-holding male. 
plates. Fluorescently labelled PCR products were resolved by electrophoresis on an ABI 3730xl capillary sequencer (Applied Biosystems, Waltham, MA, USA) and allele sizes were scored using GeneMarker v. 2.6.2 (SoftGenetics, LLC., State College, PA, USA). To maximise genotype quality, we manually inspected all of the traces and corrected any genotype calls where necessary.

\section{Quantification of the genotyping error rate}

Genotyping errors can strongly influence the outcome of genetic parentage analyses (Hoffman \& Amos, 2005; Marshall et al., 1998). We, therefore, took the precaution of independently re-extracting and re-genotyping a total of 96 randomly selected samples. The resulting duplicate genotypes were then used to calculate the error rate per genotype and per allele for each locus separately and combined over all loci.

\section{Genetic data analysis}

We removed 33 samples that were genotyped at $<15$ loci and then checked the dataset for duplicate genotypes (representing resampling events) using the $\mathrm{R}$ package 'poppr' (Kamvar et al., 2014). This identified 19 individuals that were sampled two or more times, usually in different years. Excluding these samples resulted in a final dataset of 424 unique individuals comprising 33 adult males, 101 adult females and 290 pups. We calculated the observed and expected heterozygosity of each locus using $\mathrm{R}$ package 'adegenet' (Jombart, 2008). We then tested for deviations from Hardy-Weinberg equilibrium (HWE) based on 10000 Monte Carlo permutations using the R package 'pegas' (Paradis, 2010). The resulting $P$-values were corrected using the Bonferroni correction in the p.adjust function of the 'stats' R package. Finally, we tested for population substructure by implementing a principal component analysis (PCA) of the dataset using 'adegenet'. Because PCA can be sensitive to missing data, any missing genotypes were imputed and the allele frequencies were transformed by centring and scaling the data. The probability of identity and the exclusion probability were calculated using 'GenAIEx' version 6.5 (Peakall \& Smouse, 2005).

\section{Parentage analysis}

Parentage analysis was conducted for the 290 pups within 'COLONY' 2.0.6.6 (Jones \& Wang, 2010). All sampled adult males (33 individuals) and females (101 individuals) were included as potential parents, and we did not specify any known parents or sibships. We set weak priors that the true mother and father were in the candidate lists as 0.5 and 0.2 , respectively, and specified a polygynous mating system in a diploid species. We used a medium run size, which provides high confidence parentage assignments without exceeding practicable run-times. Parentage assignments were accepted with $\geq 0.95$ probability. An advantage of COLONY is that, instead of using pairwise comparisons to assign parentage, it uses a full-pedigree maximum likelihood approach, which considers the likelihood of the entire pedigree structure and allows the simultaneous inference of both parentage and sibship. This allows the programme to assign genetically unsampled individuals as parents, which provides additional insights into mating patterns despite the incomplete sampling of parents. COLONY has been shown to be highly accurate and is the only programme available that can use genetic information to assign offspring to unsampled parents (Harrison et al., 2013; Walling et al., 2010). Wilcoxon tests were conducted in $\mathrm{R}$ base package to investigate differences in reproductive success between different categories of individual.

\section{Results}

Our final dataset for parentage analysis comprised 424 southern elephant seal individuals genotyped at 15-20 microsatellites (for details, see Table 1 and Table S1). The loci carried on average 9.3 alleles and none deviated significantly from Hardy-Weinberg equilibrium after table-wide Bonferroni correction (Table S1). The genotyping error rate, calculated by independently repeat-genotyping 96 samples, was low at 0.003 $(0.3 \%)$ per allele or $0.006(0.6 \%)$ per genotype. The probability of identity was $1.13 \times 10^{-21}$ and the exclusion probability was 1 .

\section{Parentage analysis}

COLONY assigned mothers to 108 pups and fathers to 31 pups, of which 18 had both parents assigned. Because so few pups were assigned fathers, we used two complementary approaches to analyse patterns of paternity. First, we focussed on the small number of genotyped fathers assigned to 31 pups, and second, we analysed the best configuration fathers inferred by COLONY. Best configuration fathers include genotyped fathers together with 'hypothetical genetic fathers' inferred using maximum likelihood to explain the offspring genotypes. Using the offspring genotype and the genotype of the assigned mother together with data on allele frequencies, COLONY is able to assign unsampled fathers to pups, including assigning the same unsampled father to full or paternal half siblings. Best configuration parentage assignments, therefore, allow us to investigate likely patterns of paternity, even when many fathers remain unsampled. When analysing best configuration fathers, we conservatively restricted our analysis to the 108 pups for which maternity could be assigned, thereby excluding pups with neither parent sampled.

At least one pup was assigned to $75 \%$ of genotyped females ( $n=76$ mothers) and $36 \%$ of genotyped males $(n=12$ fathers) over the course of the study (individuals with at least one pup assigned are subsequently referred to as mothers and fathers). Mothers almost exclusively had a single pup in any given year, with the exception of three pairs of twins (Fig. 3a). Annual reproductive success was significantly higher in fathers than mothers, whether limiting the analysis to genotyped fathers (Wilcoxon-test: $\mathrm{W}=543, P<0.0001$ ) or best configuration fathers (Wilcoxon-test: $\mathrm{W}=548, P=0.002$ ). Nevertheless, male reproductive skew was modest within years, with 


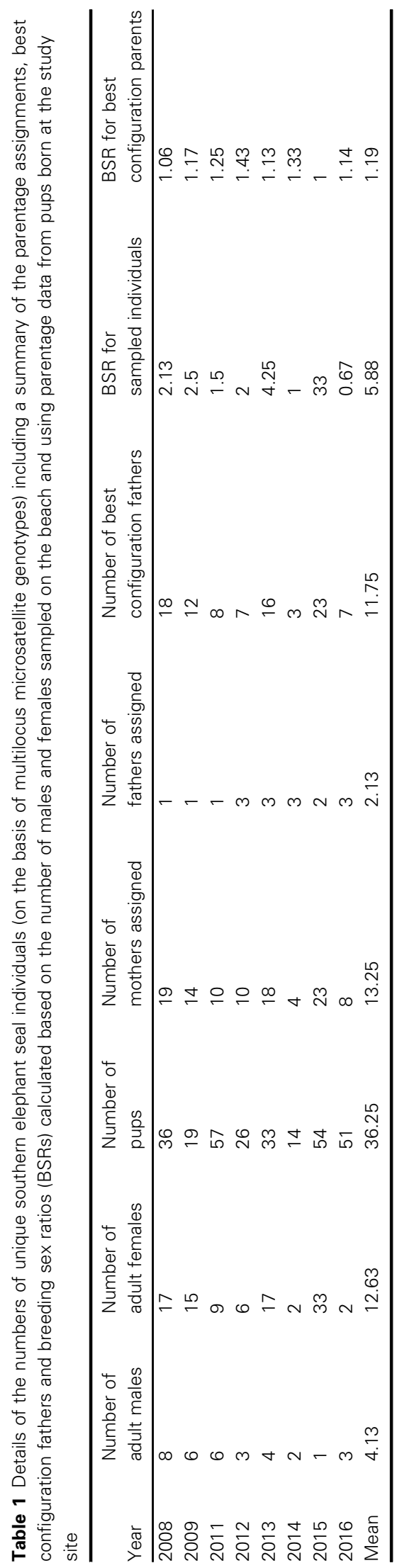

the majority of fathers only being assigned one pup $(56 \%$ of genotyped fathers, Fig. $3 \mathrm{~b}$; and $87 \%$ of best configuration fathers, Fig. 3c).

Total reproductive success over the 8 years of the study showed a qualitatively similar pattern, with genotyped fathers again producing more offspring on average than genotyped mothers (Wilcoxon-test: $\mathrm{W}=680, P=0.008$ ) although the difference was small, with mothers producing a maximum of five offspring (Fig. 3d) and fathers a maximum of seven (Fig. 3e). There was no difference in the total number of pups assigned to genotyped mothers and best configuration fathers (Wilcoxon-test: $\mathrm{W}=3012, P=0.57$, Fig. 3d,f).

The majority of individuals (50\% of genotyped fathers, $77 \%$ of best configuration fathers and $75 \%$ of genotyped mothers) only produced offspring in a single breeding season. Low reproductive skew could, therefore, result from high turnover of breeders, with many females only visiting the beach once, and hence mating with unsampled males in other locations the previous year. To investigate patterns of reproductive success in individuals that consistently bred at the study site, we identified a subset of 'core' genotyped individuals that were associated with the beach across multiple years based on genetic recaptures and parentage assignments (Fig. 4). These comprised 11 males (33\% of sampled males), 24 females ( $24 \%$ of sampled females), and the 67 pups that were genetically assigned offspring of these core adults (at least one parent was a core adult). In no year did a single male monopolise all of the offspring produced by core individuals (Table S2); the most successful male in each year fathered between $17 \%$ and $60 \%$ (mean $=32 \%)$ of these pups, equivalent to a mean of 2.6 pups per year (range $=1-5$ ). Furthermore, PCA did not identify any obvious genetic differences between core and transient individuals (Fig. 5), or between pups that were and were not assigned a father (Fig. 6), implying that they most likely originate from a single panmictic population.

Finally, although core breeders returned to the colony across multiple years, they were not always present every year. Consequently, many comparisons between candidate males and offspring will involve males who were not present in the colony during the year a given pup was conceived, while some pups may also have been conceived elsewhere if their mother was absent from the colony in the conception year. We, therefore, restricted our analysis to include only comparisons involving mothers who were known to be present in the colony during the year of conception. Although our sample size was reduced to only 20 pups, we did not find evidence for strong polygyny (Table S3). Specifically, no single male fathered more than two offspring per year, reproduction was monopolised by a single individual in only one year, and many of the pups $(35 \%)$ were fathered by unsampled males.

\section{Discussion}

Southern elephant seals have long been regarded as an example of extreme polygyny (Clutton-Brock, 2016), with 'beachmaster' males monopolising large harems of breeding females (Carrick \& Ingham, 1962) and fathering up to $90 \%$ of all offspring (Fabiani et al., 2004; Hoelzel et al., 1999; Wainstein et al., 1997). However, previous studies of this species have 


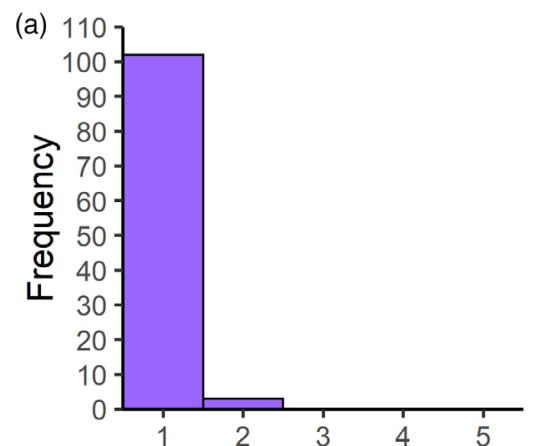

No. offspring / female / year

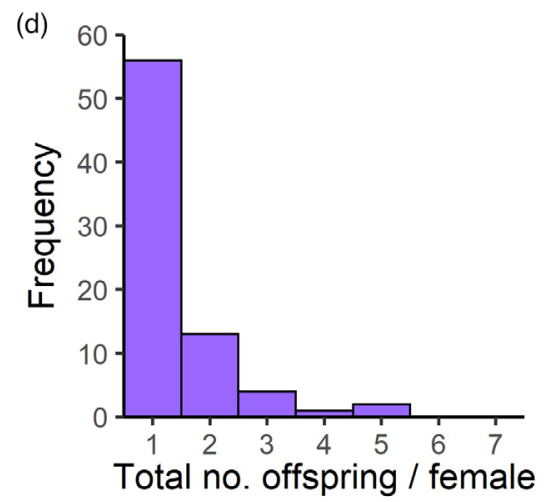

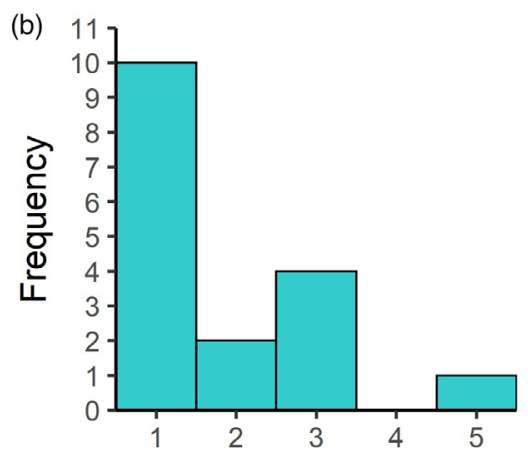

No. offspring / male / year

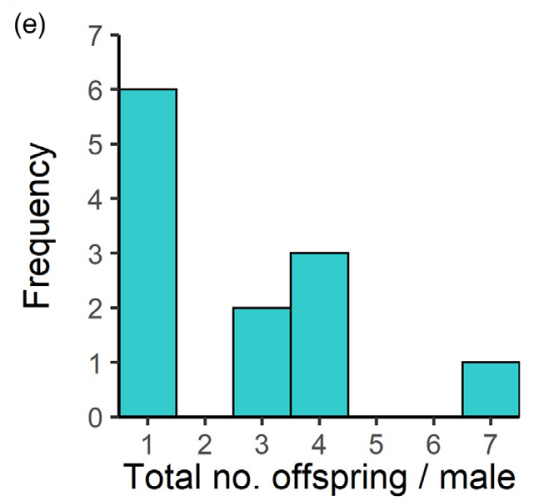

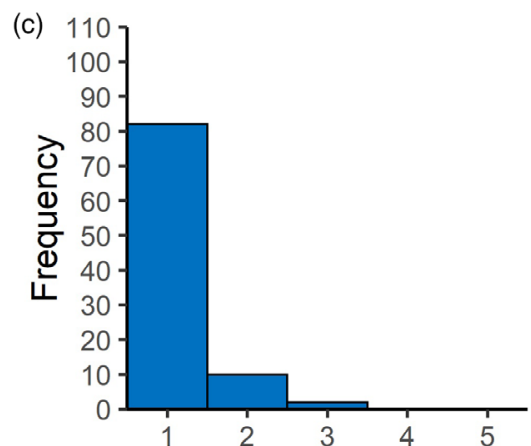

No. offspring / male / year (best configuration)

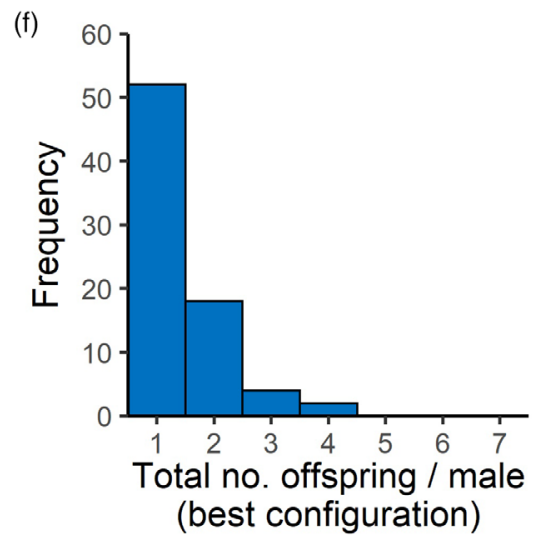

Figure 3 Reproductive skew in southern elephant seals inferred from parentage assignments (panels a,b,d,e) as well as from the best configuration fathers inferred by COLONY (panels c,f). Note that the latter was conservatively inferred from pups that were assigned maternity to a genotyped female, thereby excluding pups with neither parent sampled. The number of offspring assigned to genotyped fathers was, in some cases, larger than the number of offspring assigned to best configuration fathers because $\sim 40 \%$ of paternities were assigned to pups that were not assigned to a genotyped mother. Panels (a-c): number of offspring assigned per year of study (where the individual was assigned at least one offspring in the given year); panels (d-f): number of offspring assigned combining all years.

uncovered appreciable variation in the environmental potential for polygyny. We, therefore, investigated patterns of parentage at a southern elephant seal breeding colony in the South Shetlands, where low densities and high rates of turnover among animals may lead to different outcomes in terms of male reproductive success. We found that polygyny was relatively weak at Cape Shirreff, where paternity could only be assigned to around ten per cent of pups and reproductive skew was only slightly stronger in males than in females. We discuss these findings in the context of reproductive skew in harem-holding species and mating system flexibility, as well as in the light of specific features of the focal population.

\section{Paternity assignment rate and strength of inferred polygyny}

We were only able to assign paternity to 31 out of 290 pups $(10.7 \%)$. This low rate of paternity assignment contrasts with previous molecular genetic studies of southern elephant seals at the Península Valdés, South Georgia and the Falkland Islands, where $58 \%, 74 \%$ and $90 \%$ of paternities, respectively, were attributed to harem holders (Fabiani et al., 2004; Hoelzel et al., 1999; Wainstein et al., 1997). We also found that the strength of polygyny at the South Shetlands was much weaker than observed in similar studies of elephant seals at other localities. Specifically, the most successful males in our study were assigned up to five offspring in a single season and up to seven pups across all seasons combined. By comparison, harem holders at the Falkland Islands were assigned between 25 and 32 paternities in a single year (Fabiani et al., 2004).

These findings are surprising because, in theory, small harems should be easier for behaviourally dominant males to monopolise. Indeed, across species, larger harems have been shown to have greater rates of extra pair paternity, with the dominant males of larger harems losing out on a larger proportion of paternities (Clutton-Brock \& Isvaran, 2006; Isvaran \& Clutton-Brock, 2007), and the same may also be true within species. In the Antarctic fur seal, another pinniped that breeds in both low and high density colonies (Meise et al., 2016), dominant males appear to achieve greater reproductive success at low density (Bonin et al., 2014; Hoffman et al., 2003) and pairs of individuals repeatedly re-mate across years, implying a 


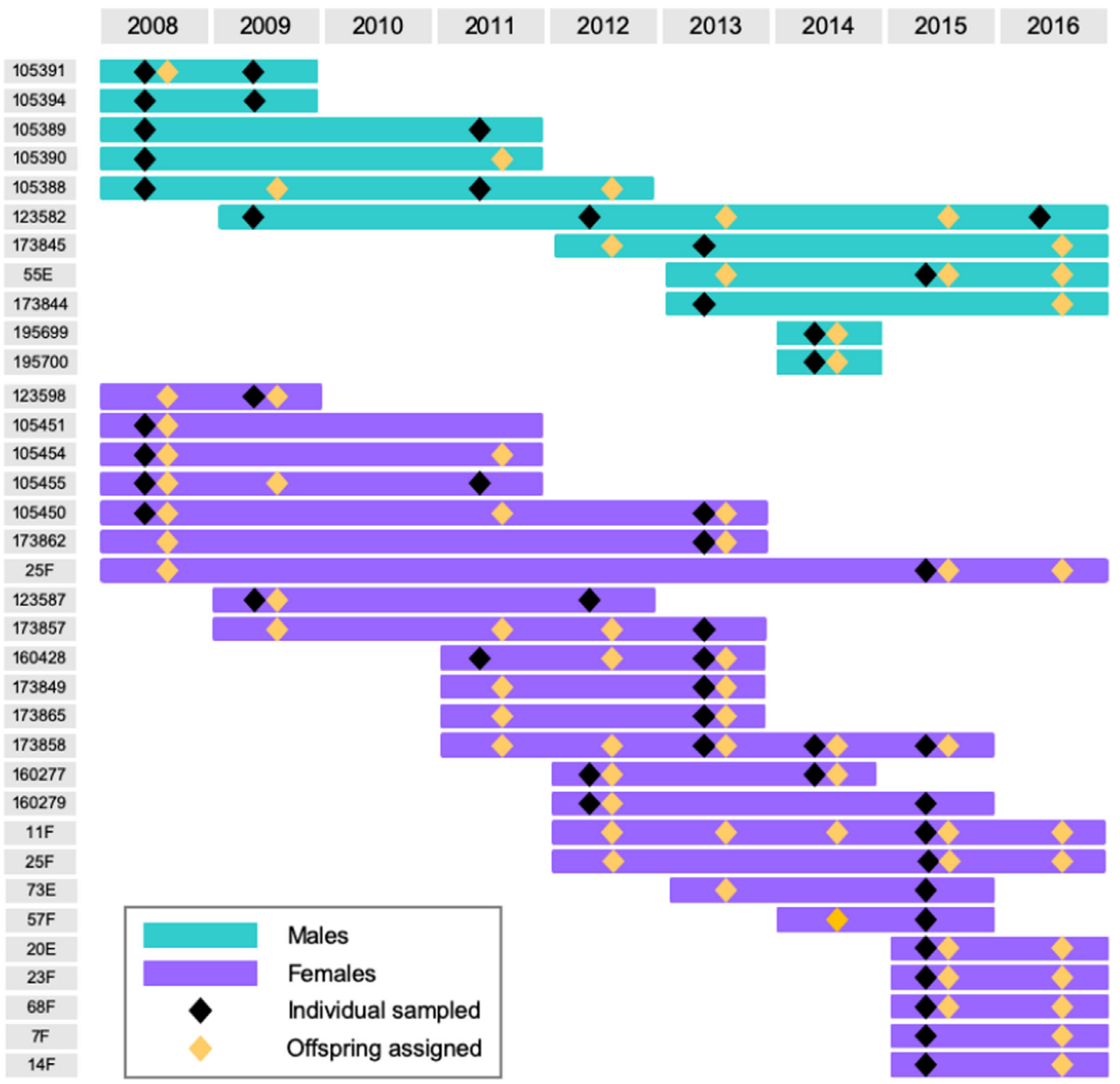

Figure 4 Breeding histories of 'core' elephant seal individuals inferred from genetic recaptures (black diamonds) and parentage assignments (yellow diamonds). Males are shown above in green and females below in purple.

relatively static mating system in which territorial males are more successful at monopolising access to breeding females. So why do southern elephant seals at Cape Shirreff deviate from the expected pattern? We can think of a number of possible explanations, broadly classified into (1) methodological aspects such as the quality of the genetic data and the completeness of sampling; (2) demographic factors such as small population size and high breeding female turnover; and (3) alternative reproductive strategies.

\section{Methodological aspects}

The importance of methodology cannot be understated in molecular genetic parentage studies. For example, even a relatively modest genotyping error rate of $1 \%$ per allele can result in over $20 \%$ of true fathers being excluded from paternity when around ten microsatellites are used (Hoffman \& Amos, 2005). We guarded against this possibility by implementing strict quality control measures including carefully standardising 

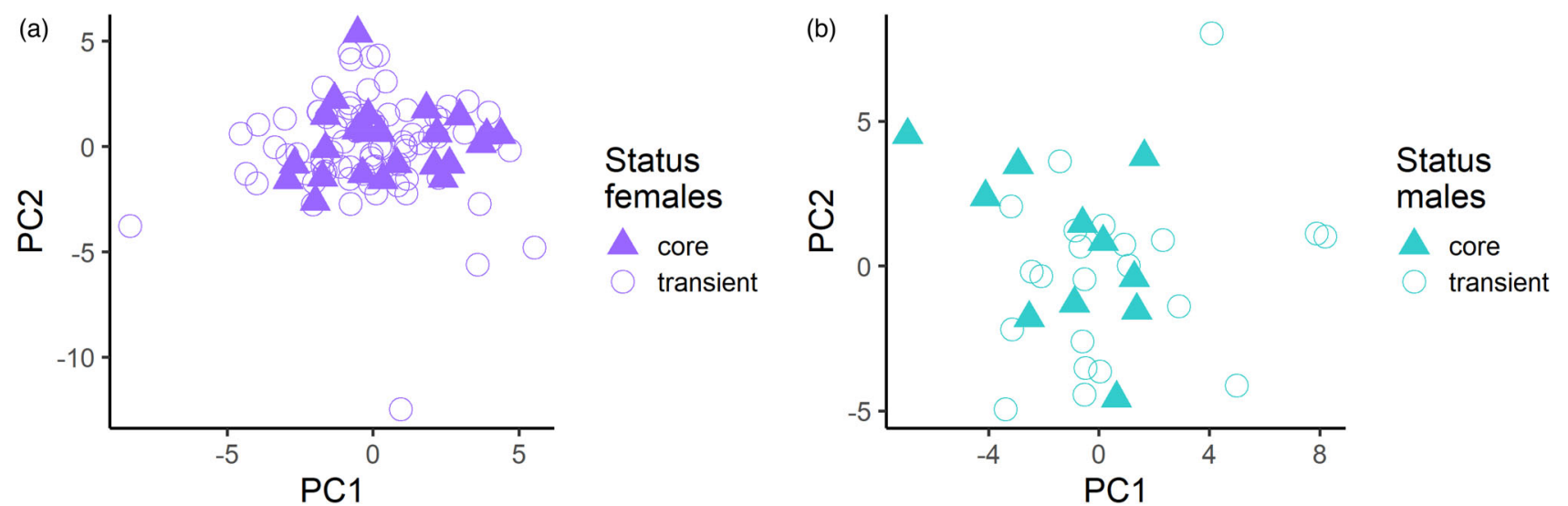

Figure 5 Results of PCAs of (a) breeding females; and (b) breeding males. The points represent individual variation in principal components one and two. Symbol-colour combinations distinguish between 'core' and 'transient' breeders as defined in the main text.

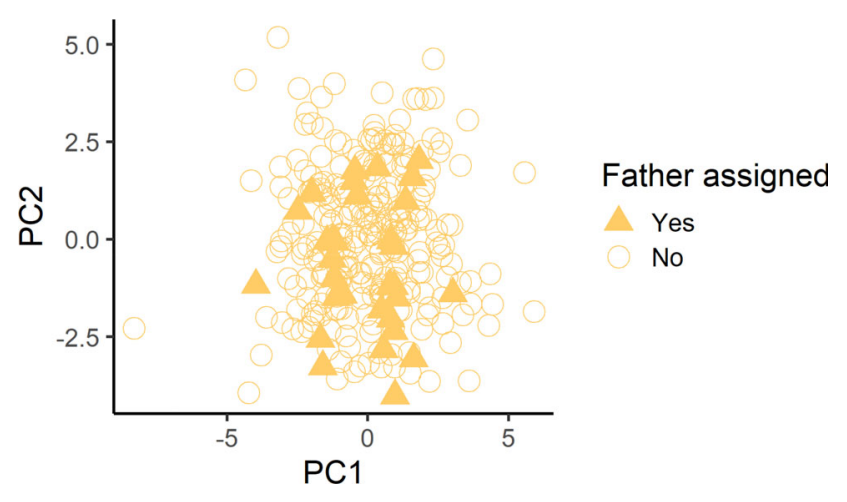

Figure 6 Results of PCA of 290 southern elephant seal pups. The points represent individual variation in principal components one and two. Symbol-fill combinations distinguish between pups assigned paternity and pups not assigned paternity.

allele scoring across plates through the inclusion of multiple positive controls, manually checking all traces and independently re-extracting and blind genotyping almost a quarter of the animals selected at random. As the resulting error rate estimate was only a fraction of a per cent, we conclude that genotyping errors are very unlikely to explain the paternity shortfall.

Previous studies of pinnipeds have also emphasised the importance of the completeness of male sampling. For example, the rate of paternity assignment in Antarctic fur seals varies from year to year in relation to the percentage of sampled males (Hoffman et al., 2003), while disparities between the outcomes of independent parentage studies of grey seals (Twiss et al., 2006; Worthington Wilmer et al., 1999) have been attributed to mismatches in the spatial and temporal coverage of male sampling. Male sampling biases could potentially have a bearing on our results given that logistical constraints prevented us from sampling throughout the entire duration of the breeding season at Cape Shirreff. Specifically, the annual sampling and marking of adults could not usually be initiated before the last week of October. We know from studies of other elephant seal colonies that female haul-out activity usually peaks sometime between the 2 nd and the 25 th of October, and that the males usually arrive around a week earlier (Galimberti \& Boitani 1999). Any males that were exclusively present in the earlier part of the season will, therefore, not have been sampled. However, our analysis of the best configuration fathers revealed a similar overall pattern to the parentage assignments and did not provide any indications of the presence of a small number of disproportionately successful males. It is, therefore, unlikely that our results can be explained by a failure to sample a small number of highly successful males. If anything, the magnitude of polygyny inferred from the best configuration fathers appeared to be slightly lower than the magnitude of polygyny inferred from paternity assignments. However, parentage analysis was performed on the full dataset while the best configuration fathers were only inferred for a subset of pups with known maternity.

\section{Demographic factors}

Our study colony of southern elephant seals at Cape Shirreff colony differs markedly from other sites where elephant seals have previously been studied. First, it is relatively small, with a mean of 36 pups produced each year in one to five harems. By contrast, population sizes and harem sizes are much larger at Sea Lion Island (mean females per harem $=47.7$, range $=18-91$; (Fabiani et al., 2004), Península Valdés (mean females per harem $=65.3$, range $=30-119$; (Campagna et al., 1993), South Georgia (mean females per harem $=74.2$, range $=6-232$; (McCann, 1980), Iles Kerguelen (mean females per harem $=76$, range 2-739; (Bester \& Lenglart, 1982) and Macquarie Island (mean females per harem $=277$, up to 1000 ; Carrick \& Ingham, 1962). The smaller harems present at Cape Shirreff may, therefore, limit the number of females that harem-holding males can monopolise, leading to reduced 
reproductive skew among males. The Cape Shirreff colony also fluctuates in size across years, with pup production varying from 14 in 2014 to 57 in 2011, whereas northerly breeding populations appear to be more stable. These fluctuations appear to be related to environmental conditions, whereby larger numbers of females breed at Cape Shirreff in warmer years, but in colder years the accumulation of sea ice and snow prevents many animals from coming ashore. As a result, around three quarters of our study females only pupped at Cape Shirreff in a single year, whereas $40-60 \%$ of females are known to return to breeding colonies at the Península Valdés over consecutive years (Hoelzel et al., 1999).

This high rate of turnover of breeding females may impact our results because many pups are likely to have been conceived at other breeding colonies, thus diluting the perceived reproductive success of the harem holders at Half Moon beach. To investigate how this might impact our results, we restricted our analysis to pups born to females that were known to be present in the colony during the year of conception. Although our sample size was substantially reduced, the overall pattern of paternity assignment was qualitatively similar and again we did not find any evidence of high male reproductive skew. Additionally, pups fathered at different colonies might be expected to carry different genetic signatures given that the four main global populations of southern elephant seals show pronounced genetic differentiation (Hoelzel et al., 2001; Slade et al., 1998). However, we could not find any obvious genetic differences between pups with known fathers and pups that were not assigned paternity. This implies that the majority of pups were probably not conceived at distant localities, although we cannot discount the possibility that females may mate at closer, less genetically differentiated sites such as elsewhere in the South Shetland Islands or at South Georgia. Movement between different subpopulations has previously been recorded in the Kerguelen population, whereby both males and females hauled out at islands other than the ones they were first observed at, with some individuals moving over $1100 \mathrm{~km}$, and there was evidence that individuals of both sexes occasionally bred at non-natal sites (Oosthuizen et al., 2011).

\section{Alternative mating strategies}

Mating might take place in or around the study colony but involve alternative reproductive strategies. For example, the unique topography of Half Moon Beach may facilitate alternative male mating strategies on land. The beach covers large areas of sandy substrate at low tide, but the animals mostly congregate around an elevated rocky area located approximately $70 \mathrm{~m}$ from the low tide line. Consequently, peripheral males only have to cross a short section of empty beach in order to access an area of high topographic complexity (rocky outcrops) that may provide sufficient cover for sneaky copulations. High complexity breeding sites with gullies and dunes appear to diminish the reproductive success of northern elephant seal (Mirounga angustirostris) harem-holding males because, even though these features concentrate females who might otherwise sparsely occupy a beach, they also provide cover for more agile peripheral males attempting to infiltrate harems to copulate (Hoelzel et al., 1999). The shape of harems may also play a role in influencing male reproductive success. For example, among southern elephant seals at Iles Kerguelen, there is a better relationship between the number of breeding males and the spatial layout of the harem than there is with the size of the harem (Bester \& Lenglart, 1982). This relationship may occur because it is more challenging for dominant males to patrol against intruders in elongated harems than in more circular harems (Bester \& Lenglart, 1982). Unfortunately, we did not measure harem shape in our study, but as all harems were relatively small, harem shape may have less impact at our study site than at Iles Kerguelen, where harems are on average much larger.

Alternatively, the large expanse of unoccupied breeding habitat at Half Moon Beach might facilitate alternative female mating strategies by making it challenging for harem-holding males to patrol against other males that would have ample opportunities for aquatic mating. Aquatic mating is relatively common in true seals and has previously been advocated as a possible explanation for the inability of molecular studies to assign paternities in grey seals (Worthington Wilmer et al., 1999) and California sea lions (Flatz et al., 2012). In addition, De Bruyn et al. (2011) argued that aquatic mating may be an important alternative female mating strategy in southern elephant seals given that breeding females often skip coming ashore yet still conceive pups. However, it is unclear whether aquatic mating may occur in females that have had the opportunity to mate on land. For example, Wilkinson and van Aarde (1999) studied 138 females breeding onshore and observed $97 \%$ of them to mate on land at least once, almost exclusively with dominant males. Beachmasters, therefore, appear capable of inseminating all females in their harems, although it is difficult to exclude the possibility that females may mate aquatically in addition to on land. Given the lack of behavioural data in our study and our inability to sample throughout the entire breeding season (researchers arrive at Cape Shirreff midway the pupping season and miss early arriving animals), we would caution against drawing premature conclusions. Nevertheless, our findings clearly point towards much lower levels of polygyny than observed at other localities (Fabiani et al., 2004; Hoelzel et al., 1999; Wainstein et al., 1997) and highlight the need for future studies focussing on both male and female reproductive strategies (De Bruyn et al., 2011).

\section{Broader perspectives}

Harem-based mating systems such as those of pinnipeds exemplify the extreme variation in reproductive success that can occur when a subset of the most behaviourally dominant males monopolise access to breeding females or the resources on which they depend (Clutton-Brock, 2016). However, a growing number of genetic studies have brought into focus the importance of alternative mating tactics such as aquatic mating, which can result in lower than expected levels of polygyny. Among polygynous pinnipeds, for example, female grey seals appear to exhibit a combination of partner fidelity (Amos 
et al., 1995) and mate choice directed towards unrelated partners (Amos et al., 2001), while overall, the proportion of offspring that can be assigned paternity is much lower than expected from observations of animals on land, implying that many females may mate at sea (Worthington Wilmer et al., 1999). Similarly, in Antarctic fur seals, California sea lions and New Zealand fur seals, female mobility (Flatz et al., 2012; Hoffman et al., 2007) and alternative male mating strategies (Caudron et al., 2010) appear to undermine polygyny. Our study contributes towards this growing body of research by showing that the variation in polygyny within a pinniped species can be greater than previously envisioned. Furthermore, our observation of weak polygyny in a low density southern elephant seal breeding colony is at odds with our original expectations based on Antarctic fur seals, where reproductive skew appears to be higher at low density (Bonin et al., 2014; Hoffman et al., 2003). This suggests that findings from one species cannot be readily extrapolated to another, and that specific features of breeding colonies such as topology, female mobility and the amount of exchange of individuals with other colonies, are likely to be important drivers of intraspecific variation in polygyny.

\section{Conclusion}

Studies of intraspecific variation in mating systems are still relatively uncommon and mating systems are often considered to be more or less fixed attributes of a given species (GurskyDoyen, 2010). However, mating systems are the outcome of the reproductive strategies of members of a species rather than evolved characteristics of a species (Clutton-Brock, 1989). Hence, they often vary in accordance with the prevailing social and ecological conditions (Bradley et al., 2005; Carranza et al., 1989; Gursky-Doyen, 2010; Jin et al., 2016; Maher \& Burger, 2011). This is exactly what we found in southern elephant seals, where pups born at Half Moon Beach in the South Shetland Islands appear to be fathered by a large number of males that each sire between one and a handful of offspring. Several factors may contribute towards this pattern, including incomplete male sampling and breeding female turnover, but these do not appear sufficient on their own to explain the relatively low reproductive success of harem holders, hinting at the possible involvement of alternative mating strategies.

\section{Acknowledgements}

All sampling was conducted in accordance with Marine Mammal Protection Act Permit Nos. 16472-01 and 774-1847-04 granted by the Office of Protected Resources, National Marine Fisheries Service, the Antarctic Conservation Act Permit Nos. 2012-005 and 2008-008. The protocols used in this study were also reviewed and approved by the U.S. National Marine Fisheries Service Southwest and Pacific Islands Region's Institutional Animal Use and Care Committee (SWR/PIR IACUC approval documents: \#SWPI2011-02, \#SWPI2014-03). We thank the following people for field assistance: Scott Freeman, Ryan Burner, Ray Buchheit, Kevin Pietrzak, Jay Wright,
Melany Zimmerman, McKenzie Mudge, Wiley Archibald, Whitney Taylor, Sam Woodman, Raul Vasquez del Mercado, David Vejar, Jessica Senzer, Michelle Goh, Matt Klosterman, Naira de Gracia, Drs. Nicola Pussini, Whitney Taylor and Douglas Krause. A special thanks to the directors of the USAMLR Program, Drs. Rennie Holt and George Watters. This work could not have been done without the support of the captain and crew of the Laurence M Gould and the National Science Foundation. We are also grateful to Laura Gerrish from the Mapping and Geographic Information Centre of the British Antarctic Survey for preparing the map. Open access funding enabled and organized by ProjektDEAL. This work was supported by the Deutsche Forschungsgemeinschaft (DFG, German Research Foundation) in the framework of a Sonderforschungsbereich (project numbers 316099922 and 396774617-TRR 212) and the priority programme 'Antarctic Research with Comparative Investigations in Arctic Ice Areas' SPP 1158 (project number 424119118). This study was also supported by core funding from the US Antarctic Marine Living Resources Program as part of their Ecosystem Monitoring Studies. HJN was supported by a Humboldt Research Fellowship for Experienced Researchers awarded by the Alexander von Humboldt Foundation. CB was funded by National Science Foundation (NSF) award number HRD 2000211 and by the National Oceanic and Atmospheric Administration Living Marine Resources Cooperative Science Center (NOAALMRCSC \# NA16SEC4810007). The funders had no role in study design, data collection and analysis, decision to publish, or preparation of the manuscript.

\section{Conflict of interests}

All authors declare no competing interests.

\section{References}

Amos, B., Twiss, S., Pomeroy, P. \& Anderson, S. (1995).

Evidence for mate fidelity in the gray seal. Science 268, 1897.

Amos, W., Worthington Wilmer, J. \& Kokko, H. (2001). Do female grey seals select genetically diverse mates? Animal Behaviour 62, 157.

Baldi, R., Campagna, C., Pedraza, S. \& Le Boeuf, B. J. (1996). Social effects of space availability on the breeding behaviour of elephant seals in Patagonia. Animal Behaviour 51, 717.

Bester, M. N. (2014). Southern elephant seal. In Hund, A. (Ed). Antarctica and the Arctic circle: A geographic encyclopedia of the Earth's polar regions. Santa Barbara, California: ABCCLIO Inc. https://books.google.co.uk/books?hl=en\&lr=\&id= wRvFBAAAQBAJ\&oi=fnd\&pg=PP1\&dq=Bester,+ M. + N . ,$+(2014)+$ Southern+elephant + seal. + In:+Antarctica + and + the + Arctic + circle: + A + geographic + encyclopedia + of + the + Earth $\%$ E2\%80\%99s + polar+regions. + A. + Hund $+($ Ed. $) .+$ ABC-CLIO+Inc. ,+Santa+Barbara.\&ots $=1 \mathrm{bHzEtFgD7 \& sig=znDoCSxYZ7s}$ zeCPTzkVGF3U7Fc\#v=onepage \&q\& $\mathrm{f}=$ false

Bester, M. \& Lenglart, P. (1982). An analysis of the southern elephant seal Mirounga leonina breeding population at Kerguelen. South African Journal of Antarctic Research 12, 11. 
Bonin, C. A., Goebel, M. E., Hoffman, J. I. \& Burton, R. S. (2014). High male reproductive skew in a low density Antarctic fur seal (Arctocephalus gazella) breeding colony. Behavioural Ecology 68, 597.

Boyd, I. L., Walker, T. R. \& Poncet, J. (1996). Status of southern elephant seals at South Georgia. Antarctic Science 8, 237.

Bradley, B. J., Robbins, M. M., Williamson, E. A., Steklis, H. D., Steklis, N. G., Eckhardt, N., Boesch, C. \& Vigilant, L. (2005). Mountain gorilla tug-of-war: Silverbacks have limited control over reproduction in multimale groups. Proceedings of the National Academy of Sciences 102, 9418.

Campagna, C., Lewis, M. \& Baldi, R. (1993). Breeding biology of southern elephant seals in Patagonia. Marine Mammal Science 9, 34.

Carranza, J., Hidalgo De Trucios, S. J. \& Ena, V. (1989). Mating system flexibility in the Great Bustard: A comparative study. Bird Study 36, 192.

Carrick, R. \& Ingham, S. E. (1962). Studies on the Southern Elephant Seal, Mirounga leonina (L.). I. Introduction to the series. CSIRO Wildlife Research 7, 89.

Caudron, A. K., Negro, S. S., Fowler, M., Boren, L., Poncin, P., Robertson, B. C. \& Gemmell, N. (2010). Alternative mating tactics in the New Zealand fur seal (Arctocephalus forsteri): when non-territorial males are successful too. Australian Journal of Zoology 57, 409.

Clutton-Brock, T. H. (1988). Reproductive success: studies of individual variation in contrasting breeding systems.

University of Chicago Press.

Clutton-Brock, T. H. (1989). Mammalian mating systems. Proceedings of the Royal Society B: Biological Sciences 236, 339.

Clutton-Brock, T. H. (2016). Mammal societies. John Wiley and Sons.

Clutton-Brock, T. H. \& Isvaran, K. (2006). Paternity loss in contrasting mammalian societies. Biology Letters 2, 513.

De Bruyn, P. J. N., Tosh, C. A., Bester, M. N., Cameron, E. Z., McIntyre, T. \& Wilkinson, I. S. (2011). Sex at sea: alternative mating system in an extremely polygynous mammal. Animal Behaviour 82, 445.

Dieckmann, U., O’Hara, B. \& Weisser, W. (1999). The evolutionary ecology of dispersal. Trends in Ecology and Evolution 14, 88.

Emlen, S. T. \& Oring, L. W. (1977). Ecology, sexual selection, and the evolution of mating systems. Science 197, 215.

Fabiani, A., Galimberti, F., Sanvito, S. \& Hoelzel, A. R. (2004). Extreme polygyny among southern elephant seals on Sea Lion Island. Falklands Islands. Behavioral Ecology 15, 961.

Fisher, R. A. (1958). The genetical theory of natural selection. Oxford University Press.

Flatz, R., González-Suárez, M., Young, J. K., HernándezCamacho, C. J., Immel, A. J. \& Gerber, L. R. (2012). Weak polygyny in California sea lions and the potential for alternative mating tactics. PLoS One 7, e33654.
Galimberti, F., Fabiani, A. \& Sanvito, S. (2002). measures of breeding inequality: A case study in southern elephant seals. Canadian Journal of Zoology 80, 1240.

Galimberti, F., \& Boitani, L. (1999). Demography and breeding biology of a small, localized population of southern elephant seals (Mirounga leonina). Marine Mammal Science 15(1), 159-178.

González-Suárez, M. \& Cassini, M. H. (2014). Variance in male reproductive success and sexual size dimorphism in pinnipeds: Testing an assumption of sexual selection theory. Mammal Review 44, 88.

Gursky-Doyen, S. (2010). Intraspecific variation in the mating system of spectral tarsiers. International Journal of Primatology 31, 1161.

Harrison, H. B., Saenz-Agudelo, P., Planes, S., Jones, G. P. \& Berumen, M. L. (2013). Relative accuracy of three common methods of parentage analysis in natural populations. Molecular Ecology 22, 1158-1170.

Hodge, S. (2009). Understanding variation in reproductive skew: directions for future empirical research. In R. Hager, \& B. J. Jones (Eds.), Reproductive skew in vertebrates: proximate and ultimate causes (p. 439). Cambridge: Cambridge University Press. https://books.google.co.uk/books?hl=en\&lr=\&id= 2bn9dL9SfxIC\&oi=fnd\&pg=PA439\&dq=Understanding + variation+in+reproductive+skew:+directions + for + future+ empirical+research\&ots=2OjYLRmwaQ\&sig=OWUYH$\mathrm{xB} 2 \mathrm{eFa} 33 \mathrm{k} 773 \mathrm{ENA} 9 \mathrm{UHOwM} \# \mathrm{v}=$ onepage $\& \mathrm{q}=$ Understanding $\%$ 20variation $\% 20 \mathrm{in} \% 20$ reproductive $\% 20$ skew $\% 3 \mathrm{~A} \% 20$ directions $\% 20$ for $\% 20$ future $\% 20$ empirical $\% 20$ research $\& \mathrm{f}=$ false

Hoelzel, A. R., Campagna, C. \& Arnbom, T. (2001). Genetic and morphometric differentiation between island and mainland southern elephant seal populations. Proceedings of the Royal Society B: Biological Sciences 268, 325.

Hoelzel, A. R., Le Boeuf, B. J., Reiter, J. \& Campagna, C. (1999). Alpha-male paternity in elephant seals. Behavioral Ecology and Sociobiology 46, 298.

Hoffman, J. I. \& Amos, W. (2005). Microsatellite genotyping errors: detection approaches, common sources and consequences for paternal exclusion. Molecular Ecology 14, 599.

Hoffman, J. I., Boyd, I. L. \& Amos, W. (2003). Male reproductive strategy and the importance of maternal status in the Antarctic fur seal Arctocephalus gazella. Evolution 57, 1917.

Hoffman, J. I., Forcada, J., Trathan, P. N. \& Amos, W. (2007). Female fur seals show active choice for males that are heterozygous and unrelated. Nature 445, 912.

Isvaran, K. \& Clutton-Brock, T. (2007). Ecological correlates of extra-group paternity in mammals. Proceedings of the Royal Society B: Biological Sciences 274, 219.

Jin, L., Yang, S. N., Liao, W. B. \& Lüpold, S. (2016). Altitude underlies variation in the mating system, somatic condition, and investment in reproductive traits in male Asian grass frogs (Fejervarya limnocharis). Behavioral Ecology and Sociobiology 70, 1197. 
Jombart, T. (2008). adegenet: a R package for the multivariate analysis of genetic markers. Bioinformatics 24, 1403.

Jones, O. R. \& Wang, J. (2010). COLONY: a program for parentage and sibship inference from multilocus genotype data. Molecular Ecology Resources 10, 551.

Kamvar, Z. N., Tabima, J. F. \& Grünwald, N. J. (2014). Poppr: an $\mathrm{R}$ package for genetic analysis of populations with clonal, partially clonal, and/or sexual reproduction. PeerJ 2, e281.

Kempenaers, B. (2008). Mate choice and genetic quality: a review of the heterozygosity theory. Advances in the Study of Animal Behaviour 37, 189.

Krzemiński, W. (1981). Southern elephant seal (Mirounga leonina L.) of Admiralty Bay (King George Island, South Shetland Islands). Its numbers and activity during the moulting season in summer. Polar Research 1, 143.

Kutsukake, N. \& Nunn, C. L. (2006). Comparative tests of reproductive skew in male primates: the roles of demographic factors and incomplete control. Behavioral Ecology and Sociobiology 60, 695.

Laws, R. M. (1956) The Elephant Seal (Mirounga leonina, Linn.): II. General, social and reproductive behaviour. Scientific Reports of the Falkland Islands Dependencies Survey, 13, 1.

Laws, R. M. (1994). History and present status of southern elephant seal populations. In R. M. Laws, \& B. J. Le Boeuf (Eds.), Elephant seals: population ecology, behavior, and physiology (p. 49). University of California Press.

Maher, C. R. \& Burger, J. R. (2011). Intraspecific variation in space use, group size, and mating systems of caviomorph rodents. Journal of Mammalogy 92, 54.

Marshall, T. C., Slate, J., Kruuk, L. E. \& Pemberton, J. M. (1998). Statistical confidence for likelihood-based paternity inference in natural populations. Molecular Ecology 7, 639.

McCann, T. S. (1980). Population structure and social organization of southern elephant seals, Mirounga leonina (L.). Biological Journal of the Linnean Society 14, 133.

McCann, T. S. (1981). Aggression and sexual activity of male southern elephant seals, Mirounga leonina. Journal of Zoology 195, 295.

McMahon, C. R., Bester, M. N., Burton, H. R., Hindell, M. A. \& Bradshaw, C. J. A. (2005). Population status, trends and a re-examination of the hypotheses explaining the recent declines of the southern elephant seal Mirounga leonina. Mammal Review 35, 82.

Meise, K., Von Engelhardt, N., Forcada, J. \& Hoffman, J. I. (2016). Maternal and offspring hormone levels reflect the prenatal social environment in fur seals: potential for fetal programming? PLoS One 11, e0145352.

Modig, A. O. (1996). Effects of body size and harem size on male reproductive behaviour in the southern elephant seal. Animal Behaviour 51, 1295.

Neff, B. D. \& Pitcher, T. E. (2005). Genetic quality and sexual selection: an integrated framework for good genes and compatible genes. Molecular Ecology 14, 19.
Nichols, D. G. (2009). Dispersal and dispersion in relation to the birthsite of the southern elephant seal, Mirounga leonina (L.), of Macquarie Island. Mammalia 34, 598.

Nunney, L. (1993). The influence of mating system and overlapping generations on effective population size. Evolution 47, 1329.

Oosthuizen, W. C., Bester, M. N., Tosh, C. A., Guinet, C., Besson, D. \& de Bruyn, P. N. (2011). Dispersal and dispersion of southern elephant seals in the Kerguelen province. Southern Ocean. Antarctic Science 23, 567.

Paradis, E. (2010). pegas: an R package for population genetics with an integrated-modular approach. Bioinformatics 26, 419.

Peakall, R. \& Smouse, P. E. (2005). GENALEX 6: genetic analysis in Excel. Population genetic software for teaching and research. Molecular Ecology Notes 6, 288.

Plesnar-Bielak, A., Skrzynecka, A. M., Prokop, Z. M. \& Radwan, J. (2012). Mating system affects population performance and extinction risk under environmental challenge. Proceedings of the Royal Society B: Biological Sciences 279, 4661.

Prokop, Z. M., Michalczyk, Ł., Drobniak, S. M., Herdegen, M. \& Radwan, J. (2012). Meta analysis suggests choosy females get sexy sons more than "good genes". Evolution 66, 2665.

Reynolds, J. D. (1996). Animal breeding systems. Trends in Ecology and Evolution 11, 68.

Ross, K. G. (2001). Molecular ecology of social behaviour: analyses of breeding systems and genetic structure. Molecular Ecology 10, 265.

Sambrook, J., Fritsch, E. F. \& Maniatis, T. (1989). Molecular cloning: A laboratory manual, 2nd ed. Cold Spring Harbour Laboratory Press.

Slade, R. W., Moritz, C., Hoelzel, A. R. \& Burton, H. R. (1998). Molecular population genetics of the southern elephant seal Mirounga leonina. Genetics 149, 1945.

Trivers, R. L. (1972). Parental investment and sexual selection. In B. Campbell (Ed.), Sexual selection and the descent of man, 1871-1971 (p. 136). Aldine-Atherton.

Twiss, S. D., Poland, V. F., Graves, J. A. \& Pomeroy, P. P. (2006). Finding fathers: spatio-temporal analysis of paternity assignment in grey seals (Halichoerus grypus). Molecular Ecology 15, 1939.

Wainstein, M. D. (2000). Paternity in southern elephant seals (Mirounga leonina). University of California.

Wainstein, M., Le Boeuf, B. J., Amos, W., Campagna, C. \& Ortiz, C. L. (1997). Mating success and paternity in the southern elephant seal (Mirounga leonina). FASEB Journal 11, 3658 .

Walling, C. A., Pemberton, J. M., Hadfield, J. D. \& Kruuk, L. E. B. (2010). Comparing parentage inference software: reanalysis of a red deer pedigree. Molecular Ecology 19, 1914-1928.

Waser, P. M., Austad, S. N. \& Keane, B. (1986). When should animals tolerate inbreeding? The American Naturalist 128, 529. 
Wilkinson, I. S. \& van Aarde, R. J. (1999). Marion Island elephant seals: the paucity of males hypothesis tested.

Canadian Journal of Zoology 77, 1547.

Worthington Wilmer, J., Allen, P. J., Pomeroy, P. P., Twiss, S. D. \& Amos, W. (1999). Where have all the fathers gone? An extensive microsatellite analysis of paternity in the grey seal (Halichoerus grypus). Molecular Ecology 8, 1417.

Zahavi, A. (1975). Mate selection-a selection for a handicap. Journal of Theoretical Biology 53, 205.

\section{Supporting Information}

Additional Supporting Information may be found in the online version of this article:

Table S1. Details of the microsatellite loci used in this study, including literature sources, polymorphism characteristics in 424 unique individuals and genotyping error rates. $H_{\mathrm{e}}$, expected heterozygosity; $H_{\mathrm{o}}$, observed heterozygosity, HWE $P$ value, uncorrected exact Hardy-Weinberg equilibrium test results based on 10000 Monte Carlo permutations.

Table S2. Details of the parentage of 'core' pups. Pups were assigned as core if either of their (genotyped) parents had produced pups on the beach in multiple years, or had been sampled in multiple years but only produced one pup.

Table S3. Results of the parentage analysis restricted to comparisons involving genotyped males and mothers who were both present in the colony during the year of pup conception. The total sample size of pups available for this analysis was 20. Colony best configuration results showed that the six pups with unsampled fathers in 2015 were fathered by five different males (one male fathered two of the pups). 